\title{
Characterisation of cell functions and receptors in Chronic Fatigue Syndrome/Myalgic Encephalomyelitis (CFS/ME)
}

Sharni Lee Hardcastle*, Ekua Weba Brenu, Samantha Johnston, Thao Nguyen, Teilah Huth, Naomi Wong, Sandra Ramos, Donald Staines and Sonya Marshall-Gradisnik

\begin{abstract}
Background: Abnormal immune function is often an underlying component of illness pathophysiology and symptom presentation. Functional and phenotypic immune-related alterations may play a role in the obscure pathomechanism of Chronic Fatigue Syndrome/Myalgic Encephalomyelitis (CFS/ME). The objective of this study was to investigate the functional ability of innate and adaptive immune cells in moderate and severe CFS/ME patients. The 1994 Fukuda criteria for CFS/ME were used to define CFS/ME patients. CFS/ME participants were grouped based on illness severity with 15 moderately affected (moderate) and 12 severely affected (severe) CFS/ME patients who were age and sex matched with 18 healthy controls. Flow cytometric protocols were used for immunological analysis of dendritic cells, monocytes and neutrophil function as well as measures of lytic proteins and T, natural killer (NK) and B cell receptors.

Results: CFS/ME patients exhibited alterations in NK receptors and adhesion markers and receptors on $C D 4^{+} T$ and $\mathrm{CD} 8^{+} T$ cells. Moderate CFS/ME patients had increased CD8 ${ }^{+}$CD45RA effector memory $T$ cells, SLAM expression on NK cells, KIR2DL5 ${ }^{+}$on $C D 4^{+} T$ cells and BTLA4 ${ }^{+}$on $C D 4^{+} T$ central memory cells. Moderate CFS/ME patients also had reduced $C D 8^{+} T$ central memory LFA-1, total CD8 ${ }^{+} T$ KLRG1, naïve CD4 ${ }^{+} T$ KLRG1 and $C D 56^{{ }^{d i m} C D 16^{-}}$NK cell CD2 ${ }^{+}$ and $\mathrm{CD} 18^{+} \mathrm{CD} 2^{+}$. Severe CFS/ME patients had increased $\mathrm{CD} 18^{+} \mathrm{CD} 11 \mathrm{C}^{-}$in the $\mathrm{CD} 56^{\mathrm{dim}} \mathrm{CD} 16^{-} \mathrm{NK}$ cell phenotype and reduced NKp46 in CD56 $6^{\text {bright }}$ CD $16^{\text {dim }}$ NK cells.
\end{abstract}

Conclusions: This research accentuated the presence of immunological abnormalities in CFS/ME and highlighted the importance of assessing functional parameters of both innate and adaptive immune systems in the illness.

Keywords: Chronic fatigue syndrome, Natural killer cell, Receptors, CD8 ${ }^{+} \mathrm{T}$ Cell

\section{Background}

The innate immune system exhibits rapid effector functions and acts as the first line of defence, protecting the host cells while activating the adaptive immune system. Natural killer (NK) cells in particular are an important interface for the innate and adaptive immune systems; hence, impaired function potentially leads to immunological disturbances. The presence of immunological dysfunction may impair physiological functioning and may play a role in disease pathogenesis [1]. NK cell dysregulation has been demonstrated in a number of illnesses,

\footnotetext{
* Correspondence: sharni.hardcastle@hotmail.com

National Centre for Neuroimmunology and Emerging Diseases, Griffith

Health Centre, School of Medical Science, Griffith University, Gold Coast, QLD,
} Australia

including human immunodeficiency syndrome (HIV), systemic lupus erythematosus (SLE), multiple sclerosis (MS) and Major Depressive Disorder (MDD) [2-4].

Patients with Chronic Fatigue Syndrome/Myalgic Encephalomyelitis (CFS/ME) exhibit reduced NK and $\mathrm{CD} 8^{+} \mathrm{T}$ cell cytotoxic activity and differences in a number of adaptive immune cell phenotypes [5, 6]. Significant decreases in NK cell cytotoxic activity in CFS/ME patients who were moderately affected by symptoms and characterised using the Fukuda criteria for the illness have been extensively reported [5, 7-23]. The reduced NK cell cytotoxic activity in CFS/ME patients may be associated with abnormalities in NK cell phenotypes, receptors or lytic proteins. Of the previous CFS/ME studies, five have also found significant differences in perforin and granzymes in NK cells of CFS/ 
ME patients [10, 20, 24-26]. These changes in functional and phenotypic components of immune cells may be playing a role in the pathomechanism of CFS/ME.

$\mathrm{CFS} / \mathrm{ME}$ is an enigmatic illness which has no known pathomechanism or cause, with diagnoses based on symptom specific criteria and a range of exclusions. Due to the multifactorial and complex nature of CFS/ME, there are often misconceptions and inconsistencies surrounding diagnosis which highlights the importance of thorough screening of participants in both clinical and research settings. This is particularly important in determining those with other illnesses such as major depression who may satisfy the CFS/ME symptom specific criteria and also demonstrate NK cell abnormalities [2]. CFS/ME is characterised by persistent fatigue and a combination of symptoms which are often severely debilitating and the illness also tends to vary greatly in the nature of onset and symptom severity [5, 7, 27-31]. Moderate patients are mostly able to maintain normal daily activities but may be hampered by reduced mobility while severely affected CFS/ME patients experience high levels of daily fatigue and are typically housebound [32].

Importantly, severe CFS/ME patients' NK cell cytotoxic activity has only been reported in three previous investigations [5, 7, 13]. Severely affected CFS/ME patients have demonstrated significant reductions in NK cell cytotoxic activity as well as increased NK cell receptor KIR3DL1 and enhanced plasma interleukin (IL)-4, tumour necrosis factor (TNF) $-\alpha$ and interferon (IFN)- $\gamma[7,13]$. This research also suggested a correlation between low NK cell cytotoxic activity and severity of CFS/ME, based on clinical status [13]. As studies have investigated innate and adaptive immune cells in CFS/ME patients, it appears important to further examine functional parameters such as cell activity, receptors and adhesion molecules. Along with NK cell and $\mathrm{CD}^{+} \mathrm{T}$ cell aberrations $[5,7,11,19,33]$, dendritic cell (DC) phenotypes have been previously abnormal in CFS/ME patients [5] although to date no study has assessed DC activity in the illness. Similarly, iNKT cells are seldom examined although one study found differences in iNKT cell phenotypes in CFS/ME patients [5], suggesting the possibility of iNKT cell dysfunction in the illness and the need to assess cytotoxic granules in these cells. Cytotoxic granules have not previously been assessed in gamma delta $(\gamma \delta)$ or regulatory T cells (Tregs) in CFS/ME patients, which may be cells of interest according to differences found in these cell types in previous research $[10,16,17,34]$.

Previous research has outlined the presence of NK cell dysfunction and immunological abnormalities in CFS/ ME patients although most studies only assessed moderately affected CFS/ME patients. Immune dysregulation can also be associated with the clinical features and aetiology of CFS/ME. This is supported as research has found significant clinical improvements in CFS/ME patients' symptoms after B cell depletion [35]. It is also possible that further immune cells may be affected by the illness and have yet to be assessed in CFS/ME patients. The current investigation was the first to measure the functional activity of DCs, neutrophils and monocytes, lytic proteins in iNKT, $\gamma \delta$ and Tregs as well as receptors and adhesion molecules of NK, T and B cells in moderate and severe CFS/ME patients.

\section{Results \\ No differences in participant characteristics between groups}

Principal participant results are reported in Table 1. We found no statistical difference for age or gender between participant groups $(p=0.325,0.607$ respectively) (Table 1 ) and the Fukuda criteria for CFS/ME were satisfied by all moderate and severe CFS/ME participants.

\section{Severity scale scores differ between participant groups}

In all severity scales used, including the Fatigue Severity Scale (FSS), Dr Bell's Disability Scale, the FibroFatigue Scale and the Karnofsky Performance Scale (KPS), there were significantly different scores between all participant groups, with the exception of 'sadness' $(p=0.064)$. Moderate CFS/ME patient scores were significantly worsened compared with healthy controls in all parameters. Severe CFS/ME patients also displayed significantly worsened scores when compared with healthy controls, with the exception of 'sadness' $(p=0.194)$ and 'sleep' $(p=0.091)$. The KPS and Dr Bell's Disability Scale scores were significantly lower in the severe CFS/ME patients compared with the moderate CFS/ME patients $(p=0.001$ and 0.001).

Table 1 Participant characteristics and comparisons of the age (mean \pm SEM) and gender distribution of each participant group (control, moderate and severe)

\begin{tabular}{lllll}
\hline Parameter & Control $(n=18)$ & Moderate $(n=15)$ & Severe $(n=12)$ & $p$ value \\
\hline Age in years (Mean \pm SEM) & $40.39 \pm 2.65$ & $45.93 \pm 2.96$ & $41.25 \pm 2.77$ & 0.325 \\
Gender Female, Male & 12,6 & 11,4 & 10,2 & 0.607 \\
\hline
\end{tabular}

Age data is represented as Mean \pm SEM in control $(n=18)$, moderate CFS/ME $(n=15)$ and severe CFS/ME $(n=12)$. *signifies $p<0.05$ between participant groups. There were no significant differences in age or gender within the research groups. CFS/ME Chronic Fatigue Syndrome/Myalgic Encephalomyelitis; standard error of the mean 


\section{Differences in routine full blood count parameters between participant groups}

Our data have shown a significantly increased monocyte count in the moderate CFS/ME patients compared with the healthy controls and severe CFS/ME patients (Fig. 1). There were no other statistically significant differences in the routine full blood count parameters between the participant groups (data not shown).

\section{No differences in flow cytometric analysis of DC, neutrophil} and monocyte function or lytic proteins

Previous research has reported differences in DC phenotypes in moderate and severe CFS/ME patients [5], however, this was the first research to assess DC activity in the illness. Our data have found no significant differences in the DC activity markers CD80 and CD86, in unstimulated or stimulated DCs between any of the participant groups, see Additional file 1: Figure S1. Neutrophil and monocyte function were examined as neutrophil respiratory burst has previously been reduced in moderate CFS/ME patients [8]. There were no significant alterations between any of the participant groups in the ability of neutrophils or monocytes to phagocytose or undergo respiratory burst, see Additional file 2: Figure S2. iNKT, $\gamma \delta$ T cells and Tregs have previously shown dysfunction in CFS/ME patients [5], however no studies had examined lytic proteins in these cell types. We found no significant differences in iNKT, $\gamma \delta \mathrm{T}$ cells or Treg levels of perforin, granzyme A, granzyme B or CD57, see Additional file 3: Figure S3.

\section{NK cell adhesion molecules and natural cytotoxicity} receptors differ between moderate and severe CFS/ME patients

Previous investigations have shown significant differences in NK cell receptors in CFS/ME patients, however signaling lymphocytic activation molecule (SLAM) receptors, adhesion molecules and natural cytotoxicity receptors have not been reported and are critical for NK cell function $[5,10]$. SLAM receptor (CD150) was significantly increased in our data in total NK cells of moderate CFS/ME patients compared with severe CFS/ME patients $(p=$ 0.046). CD56 ${ }^{\text {bright }}$ CD $16^{\text {dim }}$ NK cells expression of NKp46 was significantly reduced in severe CFS/ME compared with controls and moderate CFS/ME $(p=0.021$ and 0.021 respectively) (Fig. 2). CD56 ${ }^{\mathrm{dim}} \mathrm{CD} 16^{-} \mathrm{NK}$ cell CD2 expression was significantly lower in moderate CFS/ME compared with severe CFS/ME patients $(p=0.033)$ while $\mathrm{CD} 18^{+} \mathrm{CD} 2^{-}$was increased in moderate CFS/ME patients compared with controls and severe CFS/ME in $\mathrm{CD} 56{ }^{\mathrm{dim}} \mathrm{CD} 16^{-} \mathrm{NK}$ cells $(p=0.009$ and 0.035 respectively). In the CD $56{ }^{\mathrm{dim}} \mathrm{CD} 16^{-} \mathrm{NK}$ cells phenotype, $\mathrm{CD} 18^{+} \mathrm{CD} 11 \mathrm{c}^{-}$was significantly increased in the severe CFS/ME compared with controls $(p=0.036)$ (Fig. 2) (Table 2).

\section{No differences in Bregs and BCRs}

Significant B cell phenotypes have been reported in both moderate and severe CFS/ME patients [5], however, regulatory $\mathrm{B}$ (Breg) cells and $\mathrm{B}$ cell receptors (BCRs) in CFS/ME cohorts are yet to be examined [5, 35]. We found no significant differences in Breg cell phenotypes or BCRs between the participant groups, see Additional file 4: Figure S4.

\section{Increased KIR2DL5 in CD4 ${ }^{+} \mathrm{T}$ cells of moderate CFS/ME patients}

Killer immunoglobulin-like receptor (KIR)s have previously shown significant differences in NK cells of CFS/ ME patients, although these had not been examined in $\mathrm{CD}^{+} \mathrm{T}$ or $\mathrm{CD}^{+} \mathrm{T}$ cells in CFS/ME patients $[5,7]$. Our data found no significant alterations in the expression of KIRs on $\mathrm{CD}^{+} \mathrm{T}$ cells between the participant groups. KIR2DL5 expression was significantly higher on $\mathrm{CD}^{+} \mathrm{T}$ cells in moderate CFS/ME compared with severe CFS/ ME patients $(p=0.011)$ (Fig. 3) (Table 2).

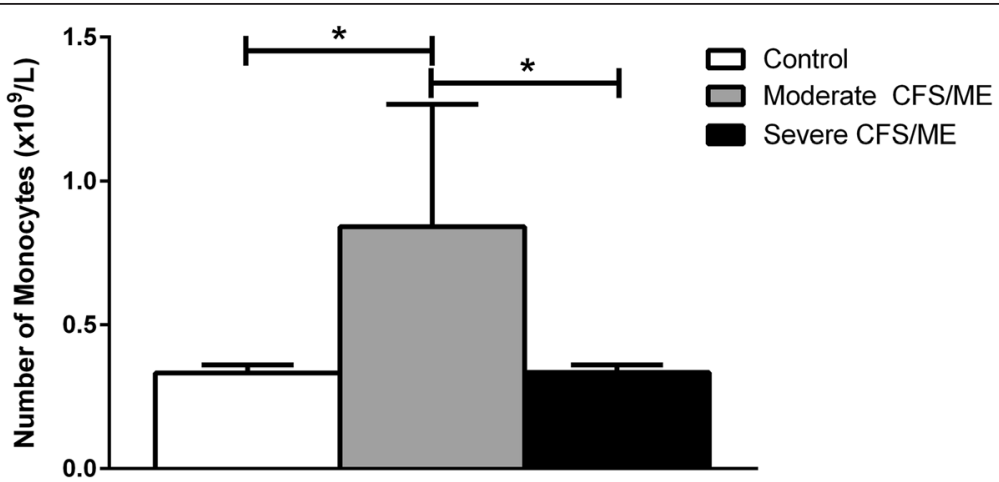

Fig. 1 Monocyte full blood counts for controls, moderate and severe CFS/ME participants. Monocyte numbers $\left(x 10^{9} / \mathrm{L}\right)$ obtained from routine full blood counts are shown for controls, moderate and severe CFS/ME patients. Data is represented as mean \pm SEM. *represents results that were significantly different where $p<0.05$. CFS/ME: Chronic Fatigue Syndrome/Myalgic Encephalomyelitis; SEM: Standard Error of the Mean 

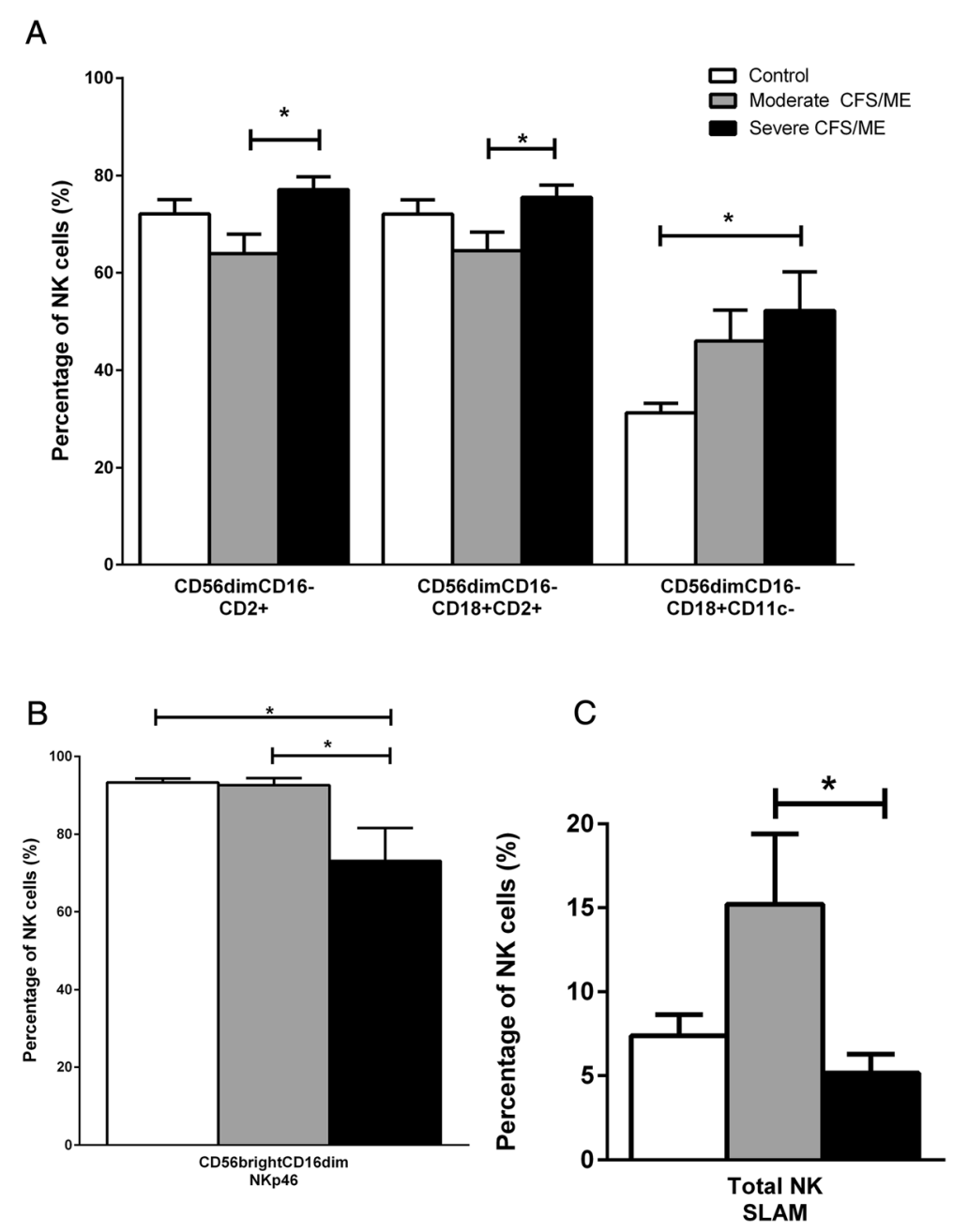

Fig. 2 The profile of receptors and adhesion molecules on isolated NK cells in controls, moderate and severe CFS/ME participants. a Profile of CD2 ${ }^{+}$, $\mathrm{CD} 18^{+} \mathrm{CD} 2^{+}$and $\mathrm{CD} 18^{+} \mathrm{CD} 11 \mathrm{c}^{-}$adhesion molecules on CD56 ${ }^{\text {dim }} \mathrm{CD} 16^{-}$NK cells in control, moderate and severe CFS/ME patients. b CD56 $6^{\text {bright }}$ CD $16^{\text {dim }}$ NK cell expression in control, moderate and severe CFS/ME patients, represented as percentage of NK cells. c Total NK cells expressing the SLAM receptor in control, moderate CFS/ME and severe CFS/ME patients. All data are represented as percentage of total NK cells (\%) and shown as mean \pm SEM. *represents results that were significantly different where $p<0.05$. NK: natural killer; CFS/ME: Chronic Fatigue Syndrome/Myalgic Encephalomyelitis; SLAM: signalling lymphocyte activation molecule; SEM: Standard Error of the Mean

\section{Differences in $\mathrm{CD} 8^{+} \mathrm{T}$ and $\mathrm{CD} 4^{+} \mathrm{T}$ cells and phenotypes between CFS/ME patient groups}

$\mathrm{CD} 8^{+} \mathrm{T}$ cells have been significantly different in CFS/ME patients in previous investigations, however, receptors on $\mathrm{CD} 8^{+} \mathrm{T}$ and $\mathrm{CD} 4^{+} \mathrm{T}$ cells had not yet been examined $[6,11,25]$. We found that the CD45RA effector memory $\mathrm{CD}^{+} \mathrm{T}$ cell phenotype formed a significantly higher percentage of total $\mathrm{CD} 8^{+} \mathrm{T}$ cells in moderate CFS/ME compared with controls $(p=0.016)$ (Fig. 4). Central memory $\mathrm{CD}^{+} \mathrm{T}$ cells had significantly reduced lymphocyte function-associated antigen (LFA) -1 in moderate CFS/ME compared with controls $(p=0.032)$. Total $\mathrm{CD}^{+} \mathrm{T}$ cell expression of killer cell lectin-like receptor subfamily $\mathrm{G}$ member (KLRG) 1 was also reduced in moderate CFS/ME compared with controls $(p=0.014)$ (Fig. 4).
In our data, $\mathrm{CD} 4^{+}$central memory $\mathrm{T}$ cells, $\mathrm{B}$ and $\mathrm{T}$ lymphocyte attenuator (BTLA)4 had significantly increased expression in moderate CFS/ME patients compared with controls $(p=0.038)$ (Fig. 3$)$. KLRG1 was also significantly reduced in $\mathrm{CD}^{+}$naïve $\mathrm{T}$ cells in moderate CFS/ME compared with controls and severe CFS/ME $(p=0.013$ and 0.019 respectively) (Table 2). There was no significant difference in $\mathrm{CD} 4^{+} \mathrm{T}$ cell phenotypes between any of the participant groups, see Additional file 5: Figure S5.

\section{Correlations across severity and immune parameters}

Our data showed a significantly positive correlation between the KPS and Dr Bell's Disability Scale scores. Both the KPS and Dr Bell's Disability scale were negatively 
Table 2 Summary of significant differences in parameters found between groups

\begin{tabular}{|c|c|c|c|}
\hline & Significant Parameters & Group & Potential Result of Changes \\
\hline \multirow[t]{5}{*}{ NK cell } & $\downarrow \mathrm{CD}_{5} 6^{\mathrm{dim}} \mathrm{CD} 16^{-} \mathrm{CD} 2^{+}$ & $M \vee S$ & Impaired ability to adhere to target cells [24]. \\
\hline & $\downarrow \mathrm{CD} 6^{\mathrm{dim}} \mathrm{CD} 16^{-} \mathrm{CD}_{18} \mathrm{CD}^{+}$ & $M \vee S$ & $\begin{array}{l}\text { Reduced NK cells in an active state hence lessened NK cell } \\
\text { activation and cytotoxic activity }[24,55] .\end{array}$ \\
\hline & $\uparrow \mathrm{CD}^{\mathrm{dim}} \mathrm{CD}^{\mathrm{d}} 6^{-} \mathrm{CD} 18^{+} \mathrm{CD} 11 \mathrm{c}^{-}$ & $\mathrm{S} \vee \mathrm{C}$ & Decreased adhesive abilities or lower number of activated cells [55]. \\
\hline & $\downarrow$ CD56 ${ }^{\text {bright }} C D 16^{\text {dim }}$ NKp46 & $S \vee C, S \vee M$ & Reduced recognition and lysis of target cells [41]. \\
\hline & $\downarrow$ Total SLAM & $S \vee M$ & Lessened ability of NK cells to undergo cytotoxic activity [40]. \\
\hline \multirow[t]{3}{*}{$\mathrm{CD}^{+} \mathrm{T}$ cell } & $\uparrow$ Total KIR2DL5 & $M \vee S$ & Inhibition of T cell functions [51]. \\
\hline & $\downarrow$ Naïve KLRG1 & $M \vee C, M \vee S$ & Enhanced T cell activation [46]. \\
\hline & $\uparrow$ Central Memory BTLA4 & $M \vee C$ & Greater inhibitory signalling and modulation of immune responses [52]. \\
\hline \multirow[t]{3}{*}{$\mathrm{CD}^{+} \mathrm{T}$ cell } & $\uparrow$ CD45RA Effector Memory & $M \vee C$ & Heightened capacity for cytotoxic activities [43]. \\
\hline & $\downarrow$ Central Memory LFA-1 & $M \vee C$ & Lack of LFA-1 adhesion required for optimal cytotoxic activity [53]. \\
\hline & $\downarrow$ Total KLRG1 & $M \vee C$ & Enhanced T cell activation [46]. \\
\hline
\end{tabular}

$\downarrow$ represents significant reductions and $\uparrow$ represents significant increases in the group listed first in the 'Group' column compared with the group listed second in the 'Group' column. S Severe CFS/ME patients; M Moderate CFS/ME patients; C Controls

correlated with the total $\gamma \delta \mathrm{T}$ cell CD45RA effector memory phenotype values and $\mathrm{CD} 56{ }^{\mathrm{dim}} \mathrm{CD} 16^{-}$NK cells with $\mathrm{CD} 18^{+} \mathrm{CD} 11 \mathrm{c}^{-}$(Table 3). There were also a number of parameters that were correlated with one another, such as $\mathrm{CD} 4^{+} \mathrm{T}$ and $\mathrm{CD}^{+} \mathrm{T}$ cell markers, NK cell adhesion markers and $\gamma \delta$ and $C D 8^{+} \mathrm{T}$ cell phenotypes (Table 3 ).

\section{Discussion}

This study is the first to provide an overview of cell function and receptor interactions, including assessing DC, neutrophil and monocyte function and receptors on T cells, BCRs and Bregs in moderate and severe CFS/ ME patients. This is also the first study to examine lytic proteins in $\gamma \delta \mathrm{T}$ cells, iNKT cells and Tregs in CFS/ME patients. Our results suggest that in some cases, functional immunological impairment may be related to differences in severity of CFS/ME patients, highlighting the variation in the illness. Significant alterations shown in moderate CFS/ME patients were often not present in severe CFS/ME patients and controls, revealing the possibility that moderate CFS/ME patients may have a different aetiology compared with the severely affected subgroup of CFS/ME patients.

Increased SLAM expression on total NK cells in moderate CFS/ME patients in this study may be a mechanistic response to the typically reduced NK cell cytotoxic activity in CFS/ME [10, 11, 36]. SLAM is a receptor expressed on the surface of T, B, NK and DC cells, functioning as an activating adaptor protein to amplify the recruitment of inflammatory cells, such as DCs, by activating IFN- $\gamma$ [37-39]. SLAM receptors regulate NK cell activity via association with SLAM associated protein (SAP) family adapters, where binding receptors are then coupled to the Src kinase FynT to evoke protein tyrosine phosphorylation signals [40]. Heightened expression of SLAM in moderate CFS/ME may enhance the ability of
NK cells to undergo cytotoxic activities [5, 7-11]. The activating receptor NKp46 is also typically involved in the recognition and lysis of target cells and was reduced in CD56 ${ }^{\text {bright }}$ CD $16^{\text {dim }}$ NK cells of severe CFS/ME patients [41]. NKp46 is highly expressed on the CD56 ${ }^{\text {bright }} \mathrm{CD} 16^{\text {dim }}$ NK cell subset and although it is only weakly involved in cytotoxic activation, this reduction may contribute to the reduced NK cell cytotoxic activity prevalent in severe CFS/ME patients [5, 41, 42]. The expression of SLAM and NKp46 on NK cells significantly differed between moderate and severe CFS/ME patients, suggesting that perhaps these severity subgroups may vary in immunological presentation, as proposed by previous studies $[5,7,13]$.

Effector memory and CD45RA effector memory cells demonstrate NK-like functions as they have the ability to detect abnormal major histocompatibility complex (MHC) expression and have a high capability for cytotoxic activities [43]. Our findings suggest that the number of CD45RA effector memory $\mathrm{CD}^{+} \mathrm{T}$ cells of moderate CFS/ ME patients may be enhanced due to sub-optimal function. In T cells, CD45RA effector memory cells are upregulated following cytokine directed proliferation, indicating that the subset is generated via homeostasis rather than antigen-dependent pathways [44, 45]. Moderate CFS/ME patients have an increased number of $\mathrm{CD}^{+} \mathrm{T} C \mathrm{CD} 45 \mathrm{RA}$ effector memory cells, potentially as a result of homeostasis where the cells may not be effectively undergoing degranulation and apoptosis $[44,45]$.

This research also found reductions in KLRG1 expression in total $\mathrm{CD}^{+} \mathrm{T}$ and naïve $\mathrm{CD} 4^{+} \mathrm{T}$ cells of moderate CFS/ME patients, which suggests that these cells may have a reduced ability to inhibit $\mathrm{T}$ cell function and activation. KLRG1 ligation inhibits the nuclear factor of activated $\mathrm{T}$ cells (NFAT) signalling pathway and downregulates CD95 mediated lysis to inhibit the activation of $\mathrm{T}$ cells [46]. Hence, blockades of inhibitory receptors tend to improve 


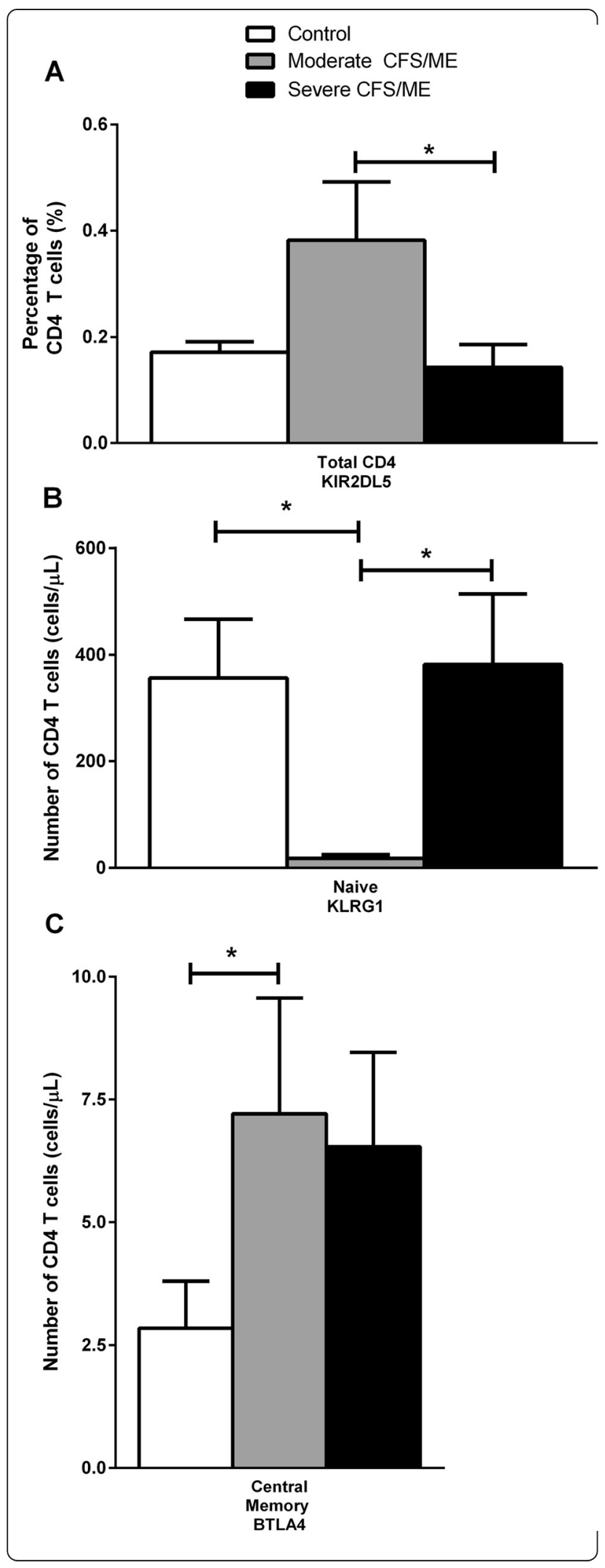

Fig. $3 \mathrm{KIR}$ and receptor expression in $\mathrm{CD} 4^{+} \mathrm{T}$ cells of controls, moderate and severe CFS/ME participants. a KIR2DL5 expression in total CD4 ${ }^{+} T$ cells as represented by a percentage of total $C D 4^{+} T$ cells (\%). b Number of naïve $C D 4^{+} T$ cells expressing KLRG1 in in control, moderate and severe CFS/ME participants shown as a number of cells per microliter (cells/ $\mu L)$. C BTLA4 receptor expression in central memory CD4 ${ }^{+} T$ cells of control, moderate and severe CFS/ME patients, presented as cells $/ \mu \mathrm{L}$. Data is represented as mean $\pm S E M$. *represents results that were significantly different where $p<0.05$. KIR: killer immunoglobulin-like receptor; CFS/ME: Chronic Fatigue Syndrome/Myalgic Encephalomyelitis; KLRG1: killer cell lectin-like receptor subfamily G member 1; BTLA4: B and T lymphocyte attenuator SEM: Standard Error of the Mean

$\mathrm{CD} 8^{+} \mathrm{T}$ cell responses by preventing inhibitory pathways [47]. It is therefore possible that reduced KLRG1 may be contributing to the pro-inflammatory response and T cell activation often found in CFS/ME patients [10, 48].

Increased KIR2DL5 on CD4 ${ }^{+} \mathrm{T}$ cells in moderate CFS/ ME patients may also be associated with alterations in KIR receptors in T cells in the same cohort [5, 7]. KIR2DL5 is an inhibitory KIR found in variable proportions of circulating $\mathrm{T}$ cells $[49,50]$ which is directly linked to a greater number of random combinations of KIR receptors expressed on these cells, which may be influencing optimal $\mathrm{T}$ cell functions in the illness [51]. Enhanced inhibitory signalling and modulation of immune responses are typical attributes of increased BTLA expression in T cells which may be present in moderate CFS/ME patients who have amplified expression of inhibitory receptor BTLA4 in central memory $\mathrm{CD}_{4}^{+} \mathrm{T}$ cells [52]. Activation and function of $\mathrm{CD}^{+} \mathrm{T}$ cells by NK cells is dependent on the engagement of the $\beta_{2}$ integrin LFA-1. LFA-1 adhesion is necessary for optimal cytotoxic activity by both $\mathrm{NK}$ cells and $\mathrm{CD} 8^{+} \mathrm{T}$ cells, also mediating NK cell degranulation via synergy with NKG2D [53]. Decreased expression of LFA-1 on central memory $\mathrm{CD}^{+} \mathrm{T}$ cells in moderate $\mathrm{CFS} / \mathrm{ME}$ patients suggests that there may be a lack of LFA-1 adhesion in CFS/ME which is required for ideal cytotoxic activity by NK cells and $\mathrm{CD}^{+} \mathrm{T}$ cells [53]. Similar to the pattern shown in SLAM and NKp46 receptors on NK cells, CD45RA effector memory $\mathrm{CD} 8^{+} \mathrm{T}$ cells and $\mathrm{CD} 4^{+} \mathrm{T}$ and $\mathrm{CD}^{+} \mathrm{T}$ cell receptors significantly differed between moderate and severe CFS/ME patients.

Cellular adhesion may be important in CFS/ME as it is required for target cell contact and NK cell effector function. Regulation of adhesion molecules is necessary for integrin target cell ligand interactions as the release of adherence results in lymphocyte movement [54]. CD2 expression in $\mathrm{CD} 56{ }^{\mathrm{dim}} \mathrm{CD} 16^{-} \mathrm{NK}$ cells is reduced in moderate CFS/ME patients compared with severely affected patients, suggesting that these cells may have an impaired ability to adhere to target cells. This confirmed previous findings where $\mathrm{CD} 2 / \mathrm{CD} 18$ co-expression was reduced in the same $\mathrm{CD} 56^{\mathrm{dim}} \mathrm{CD} 16^{-}$NK cell phenotype 

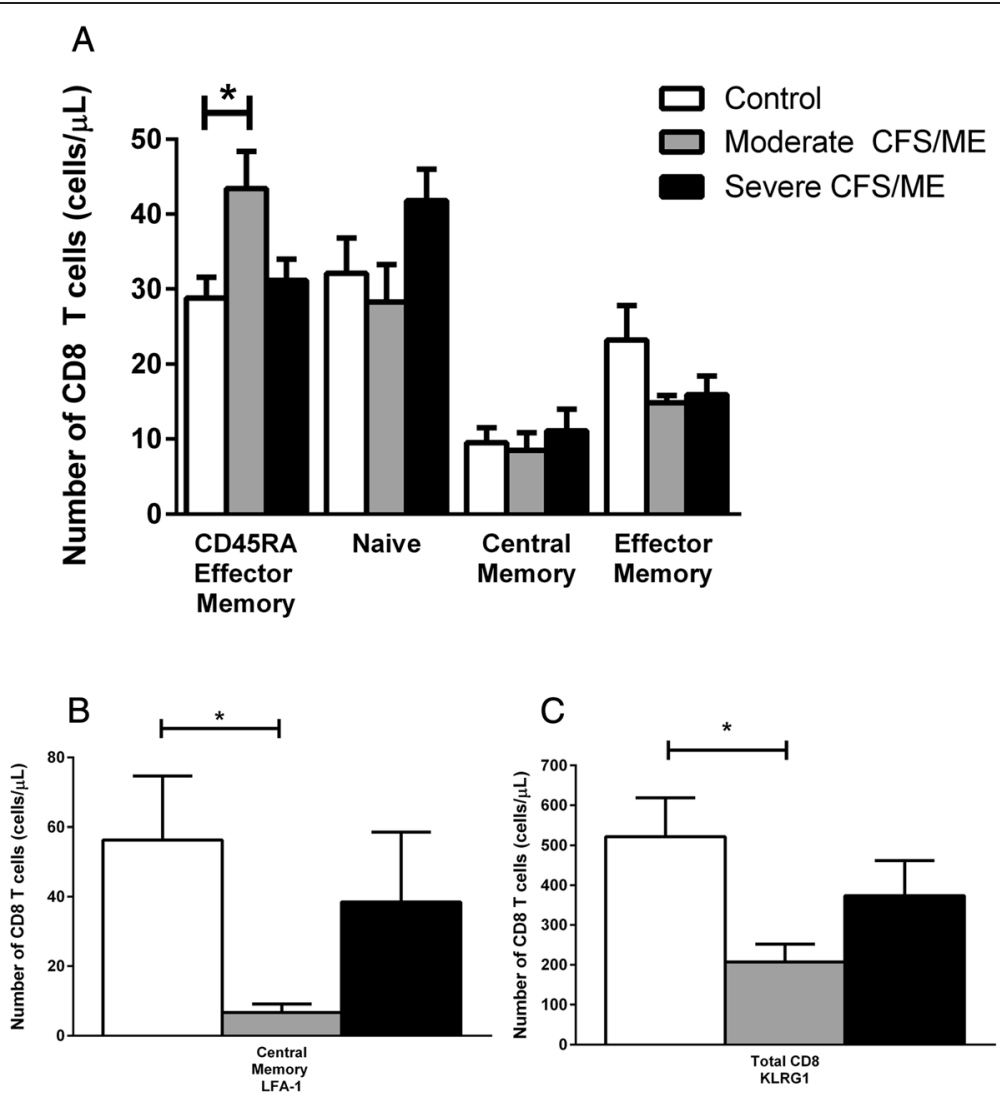

Fig. 4 Alterations in peripheral blood CD8 ${ }^{+} T$ cell phenotypes and receptors in controls, moderate and severe CFS/ME. a CD45RA effector memory, naïve, central memory and effector memory $C D 8^{+} T$ cell phenotypes represented as percentage of total $C D 8^{+} T$ cells (\%). $\mathbf{b} L F A-1$ expression in central memory CD8 ${ }^{+} T$ cells for control, moderate CFS/ME and severe CFS/ME participants. c KLRG1 expression in total CD8 ${ }^{+} T$ cells for control, moderate CFS/ ME and severe CFS/ME participants. All data are represented as mean \pm SEM. ${ }^{*}$ represents results that were significantly different where $p<0.05$. CFS/ ME: Chronic Fatigue Syndrome/Myalgic Encephalomyelitis; LFA-1: lymphocyte function-associated antigen 1; KLRG1: killer cell lectin-like receptor subfamily G member 1; SEM: Standard Error of the Mean

Table 3 Spearman's correlation to identify correlates between significant parameters

\begin{tabular}{|c|c|c|c|c|c|c|c|c|c|c|c|c|c|c|c|}
\hline & & 1 & 2 & 3 & 4 & 5 & 6 & 7 & 8 & 9 & 10 & 11 & 12 & 13 & 14 \\
\hline 1 & CM CD8 ${ }^{+}$LFA-1 & & & & & & & & & & & & & & \\
\hline 2 & Naïve $\mathrm{CD}^{+}{ }^{+} \mathrm{KLRG} 1$ & & & & & & & & & & & & & & \\
\hline 3 & Total CD8 ${ }^{+}$KLRG1 & & .50 & & & & & & & & & & & & \\
\hline 4 & $\mathrm{CM} \mathrm{CD4} 4^{+}$BTLA4 & .55 & & & & & & & & & & & & & \\
\hline 5 & $\mathrm{CM} \mathrm{CD4} 4^{+}$LFA-1 & & & .56 & & & & & & & & & & & \\
\hline 6 & $\mathrm{CD}_{5}{ }^{\mathrm{dim}} \mathrm{CD} 16^{-} \mathrm{NK} \mathrm{CD} 2^{+}$ & & & & & & & & & & & & & & \\
\hline 7 & $\mathrm{CD}_{56}{ }^{\mathrm{dim}} \mathrm{CD} 16^{-} \mathrm{NK} \mathrm{CD} 18^{+} \mathrm{CD} 2^{+}$ & & & & & & -.60 & & & & & & & & \\
\hline 8 & $\mathrm{CD} 56^{\mathrm{dim}} \mathrm{CD} 16^{-} \mathrm{NK}$ CD18 $8^{+} \mathrm{CD} 11 \mathrm{a}^{-}$ & & -.47 & & .49 & & -.57 & & & & & & & & \\
\hline 9 & $\mathrm{CD} 56^{\operatorname{dim}} \mathrm{CD} 16^{-} \mathrm{NK}$ CD $18^{+} \mathrm{CD} 11 \mathrm{C}^{-}$ & & & & & & & & & & & & & & \\
\hline 10 & Total NK SLAM & & & .46 & & & -.53 & & & -.50 & & & & & \\
\hline 11 & Total CD8 ${ }^{+}$EMRA & & & -.44 & & -.75 & & & & & & & & & \\
\hline 12 & $\mathrm{CD}^{+}{ }^{+} \mathrm{KIR} 2 \mathrm{DL} 5$ & & & & & & & & .48 & & .75 & & & & \\
\hline 13 & KPS & & & & & & & & & -.43 & & & & & \\
\hline 14 & Dr Bell's Disability Scale & & & & & & & & & -.46 & & & & .95 & \\
\hline
\end{tabular}

Table 3 shows the significant correlation values between bivariate parameters for significant parameters for combined control, moderate CFS/ME and severe CFS/ ME participant groups. All correlations shown have $p<0.01$. CM: Central Memory, EMRA: CD45RA Effector Memory 
in a cohort of moderate CFS/ME patients [24]. Increased CD2 is often associated with a higher cytotoxic ability [55] as CD2 acts as a contributor to induce NK cell activation $[54,55]$. Higher expression of CD2 in severe CFS/ ME patients potentially implies that more NK cells in these patients are in an active state and may have a greater ability than the moderate CFS/ME patients to induce NK cell activation and cytotoxic activities [55]. $\mathrm{CD} 18^{+} / \mathrm{CD} 2^{-} \mathrm{CD} 56{ }^{\mathrm{dim}} \mathrm{CD} 16^{-}$NK cells were also increased in the moderate CFS/ME patients, strengthening the theory that CFS/ME patients may have a weakened ability to activate NK cells as well as having impaired NK cell cytotoxic activity. Adhesion molecules CD18 and $\mathrm{CD} 2$ on $\mathrm{CD} 56{ }^{\mathrm{dim}} \mathrm{CD} 16^{-}$NK cells were also significantly altered in the moderate CFS/ME patient group compared with the severe CFS/ME patients, who appeared similar to the controls. In the case of $\mathrm{CD} 18^{+} \mathrm{CD} 11 \mathrm{c}^{-}$on the same $\mathrm{CD} 56^{\mathrm{dim}} \mathrm{CD} 16^{-}$NK cell subset, however, increases in $\mathrm{CD} 18^{+} \mathrm{CD} 11 \mathrm{c}^{-}$increased in the moderate CFS/ $\mathrm{ME}$ patients and significantly increased in the severe CFS/ME patients. Expression of the adhesion marker CD11c is heterogeneous and variable in NK cells although, typically activated NK cells are CD11c ${ }^{+}$[55]. Increased $\mathrm{CD} 18^{+} \mathrm{CD} 11 \mathrm{c}^{-}$on $\mathrm{CD} 56^{\mathrm{dim}} \mathrm{CD} 16^{-} \mathrm{NK}$ cells in severe CFS/ME patients indicates that these patients may have a reduced ability to adhere or that they may have a low number of activated NK cells. Therefore, differences in $\mathrm{CD} 18^{+} \mathrm{CD} 11 \mathrm{c}^{-}$adhesion molecules in severe CFS/ME patients may be associated with the reduced NK cell cytotoxic activity found in the illness $[5,10,11]$.

CFS/ME symptom severity and presentation may be related to the immune dysregulation shown as the immune system interacts with physiological functioning via a number of body systems, including the central nervous system, digestive system and endocrine system [56]. Unrefreshing sleep and sleep disturbances are symptoms of CFS/ME and reports have indicated that NK cells are altered after sleep deprivation, demonstrating interactions between physiological symptoms and the immune system [57], particularly in CFS/ME patients. Similarly, it has previously been suggested that clinical severity status appears to be associated with reduced NK cell activity in CFS/ME patients [7, 13]. Although there are limited research findings for severe CFS/ME patients, the differences in NK cells, $\mathrm{CD} 4^{+} \mathrm{T}$ and $\mathrm{CD}^{+} \mathrm{T}$ cells between severity groups, found in this research, suggest that immune dysfunction in CFS/ME may be related to clinical symptoms and hence severity.

\section{Conclusions}

This study was the first to show significant differences in a number of receptors in $\mathrm{NK}, \mathrm{CD} 4^{+} \mathrm{T}$ and $\mathrm{CD} 8^{+} \mathrm{T}$ cells in CFS/ME $[5,7]$ suggesting dysregulation in NK cell cytotoxic activity, receptor regulation and potentially cell adherence. Consistent with previous literature, our research suggests that CFS/ME patients have immunological dysregulation in the innate and adaptive immune cells. We have also highlighted significant differences in NK, $\mathrm{CD}_{4}^{+} \mathrm{T}$ and $\mathrm{CD}^{+} \mathrm{T}$ cells between moderate and severe CFS/ME patients, suggesting severity subgroups may have distinct immune perturbations and consequently aetiology. Further studies examining severity subgroups of CFS/ME patients may therefore contribute to the understanding of the pathomechanism associated with the illness.

\section{Methods \\ Participants}

Participants aged between 20 and 65 years old were recruited from Queensland and New South Wales areas of Australia through CFS/ME support groups, email advertisements and social media. In the absence of a diagnostic test for CFS/ME, the 1994 Fukuda criteria were used and patients must have had the illness for a period of at least 6 months prior to the study. All CFS/ME patients had been diagnosed by a primary physician in order to take part in the research. CFS/ME patients were identified as either moderate (mobile) or severe (housebound). These severity groups were then confirmed using an extensive questionnaire which included FSS, Dr Bell's Disability Scale, the FibroFatigue Scale and the KPS as determinants of severity. Participants were then excluded if they were previously diagnosed or had a history of an autoimmune disorder, MS, psychosis, major depression, heart disease or thyroid-related disorders or if they were pregnant, breast feeding, smokers, or experiencing symptoms of CFS/ME that did not conform to the Fukuda criteria for CFS/ME [5]. The questionnaire obtained detailed information regarding the onset of illness, presence, frequency and severity of symptoms, comorbidities, overall health and quality of life. This allowed an analysis of all participants on an individual basis to ensure there were no confounding factors influencing CFS/ME patient symptoms. In order to identify psychological exclusions in participants that were not specifically outlined, the questionnaire (including the FSS, SF-36 and WHO DAS2.0) also included questions and scales regarding emotional stability, social interaction, motivation and wellbeing $[58,59]$.

Participants $(n=45)$ included in the study were patients moderately $(n=15)$ or severely $(n=12)$ affected by CFS/ME symptoms as well as a healthy non-fatigued control group $(n=18)$. All participant groups were matched for age and sex. All CFS/ME patients had the illness for at least 6 months prior to their participation in the research and the average duration of illness of a CFS/ME patient was 6.5 years. It was then ensured that all participating CFS/ME patients fulfilled the Fukuda criteria for CFS/ME 
at the time of blood collection according to questionnaires which assessed their symptoms in the 30 days prior and at the time of collection. Written informed consent was obtained from all participants and all research protocols were granted ethical clearance after review by the Griffith University Human Research Ethics Committee (GU Ref No: MSC/23/12/HREC).

\section{Sample preparation and routine measures}

Blood collection occurred between 8:00 and 11:30 am and samples were analysed within $12 \mathrm{~h}$. A non-fasting blood sample of $50 \mathrm{~mL}$ was collected into lithium heparinised and ethylenediaminetetraacetic acid (EDTA) tubes from the antecubital vein of all participants. Initial full blood count results were determined by Pathology Queensland to assess routine levels of white blood cell and red blood cell markers.

\section{Lytic proteins analysis}

Lytic proteins were assessed as previously described [7, 10] in Tregs, iNKT, $\gamma \delta 1$ and $\gamma \delta 2$ T cells. Ficoll-hypaque (Sigma, St Louis, MO) density gradient centrifugation was used to isolate peripheral blood mononuclear cells (PBMCs) from EDTA whole blood. PBMCs were adjusted to $1 \times 10^{7}$ cells $/ \mathrm{mL}$ and stained with monoclonal antibodies for iNKT, Tregs, $\gamma \delta 1$ and $\gamma \delta 2$ T cells, see Additional file 6: Table S1. Cells were incubated for $30 \mathrm{~min}$ in Cytofix then perforin, granzyme A and granzyme B monoclonal antibodies were added for $30 \mathrm{~min}$. Cells were analysed on the flow cytometer where perforin, granzyme A and granzyme B expression was measured in iNKT, Tregs, $\gamma \delta 1$ and $\gamma \delta 2$ T cells, see Additional file 6: Table S1.

\section{DC cell activity analysis}

DC activity was measured from 300uL lithium heparinised whole blood, incubated in Roswell Park Memorial Institute (RPMI)-1640 culture media (Invitrogen, Carlsbad, CA), Phorbol 12-myristate 13-acetate (PMA) and Ionomycin for $5 \mathrm{~h}$ at $37^{\circ} \mathrm{C}, 5 \% \mathrm{CO}_{2}$. Cells were labelled with monoclonal antibodies (see Additional file 6: Table S1) and FACS Lyse (BD Biosciences, San Diego, CA) was used to remove red blood cells. Flow cytometric analysis (Becton Dickinson Immunocytometry Systems) was used to measure DC activity based on unstimulated versus stimulated assessments.

\section{Phagocytosis analysis}

The Phagotest kit was used to determine leukocyte phagocytosis in whole blood based on the ability of neutrophils and monocytes to engulf bacteria (Orpegen Pharma, Germany). Manufacturer's instructions were followed for the procedure. Lithium heparinised whole blood (100uL) was aliquot into two $5 \mathrm{~mL}$ tubes as control and test samples. Control and test samples were incubated with E.coli bacteria (Orpegen Pharma, Germany) on ice or at $37{ }^{\circ} \mathrm{C}$ respectively, before quenching solution were added to stop phagocytosis. Samples were washed and red blood cells were lysed with lysing solution (Orpegen Pharma, Germany). DNA staining solution was used as a measure of neutrophil and monocyte phagocytosis based on differential gating on a flow cytometric analysis (Becton Dickinson Immunocytometry Systems).

\section{Respiratory burst analysis}

Respiratory burst analysis was measured in granulocytes from whole blood. Intracellular oxidation was performed by incubating $100 \mathrm{uL}$ lithium heparinised whole blood in Dihydrohodamine (DHR) for $10 \mathrm{~min}$ at $37^{\circ} \mathrm{C}$. Phorbol 12-myristate 13-acetate was then added, followed by $10 \mathrm{~min}$ incubation at $37^{\circ} \mathrm{C}$. FACS Lyse (BD Biosciences, San Diego, CA) was used to remove red blood cells and DHR was used as a measure of neutrophil and monocyte respiratory burst using differential gating on the flow cytometer (Becton Dickinson Immunocytometry Systems).

\section{Isolated NK cell receptor analysis}

As previously described [5], NK cells were isolated from whole blood cells using negative selection with RosetteSep Human Natural Killer Cell Enrichment Cocktail (StemCell Technologies, Vancouver, BC). Isolated NK cells were labelled with CD56, CD16, CD3 (BD Biosciences, San Diego, CA) and monoclonal antibodies for SLAM, integrin and natural cytotoxicity receptors (NCRs), see Additional file 6: Table S1 (Miltenyi Biotec). Analysis was undertaken on the flow cytometer (Becton Dickinson Immunocytometry Systems), where NK cells were gated using CD56, CD16 and CD3 and SLAM, integrin and NCR receptors were assessed for each NK cell phenotype $\left(\mathrm{CD} 56^{\mathrm{dim}} \mathrm{CD} 16^{+}, \mathrm{CD} 56^{\text {bright }} \mathrm{CD} 16^{+}, \mathrm{CD} 56^{\mathrm{dim}} \mathrm{CD} 16^{-}\right.$and $\mathrm{CD} 6^{\text {bright }} \mathrm{CD} 16^{\text {dim }}$ ) see Additional file 6: Table S1 [7].

\section{T and B cell whole blood analysis}

Whole blood analysis was undertaken as previously described [5]. Monoclonal antibodies were added to lithium heparinised whole blood samples and incubated for 30 min, see Additional file 6: Table S1. Cells were then lysed, washed and fixed. $\mathrm{CD} 4^{+} \mathrm{T}$ and $\mathrm{CD} 8^{+} \mathrm{T}$ cell KIR receptors and phenotypes, $\mathrm{B}$ cell receptors and $\mathrm{B}$ regulatory cells were assessed using appropriate antibodies (see Additional file 6: Table S1) and gating strategies on the flow cytometer (Becton Dickinson Immunocytometry Systems) [60].

\section{Statistical analysis}

Data were compared among the three participant groups (control, moderate CFS/ME and severe CFS/ME) with statistical analysis performed based on the distribution. Shapiro-Wilk normality tests were performed and if 
normally distributed, the analysis of variance test (ANOVA) was used. If data was not normally distributed, the Kruskal Wallis test of independent variables based on rank sums to determine the magnitudes of group differences was used. The Bonferroni Post Hoc or Mann-Whitney U tests determined $p$ values of significance for parametric and non-parametric data respectively, with statistical significance set at an alpha criterion at $p<0.05$. Spearman's non-parametric correlation was conducted on significant parameters to determine correlates where significance was accepted as $p<0.01$. Outliers were identified using a boxplot technique and handled by eliminating particular extreme data points from the analysis [61].

SPSS statistical software version 22.0 was used for all statistical analysis and data represented in this study are reported as plus/minus the standard error of the mean $( \pm$ SEM) or plus/minus the standard deviation (SD) as specified.

\section{Additional files}

Additional file 1: Figure S1. Flow cytometric analysis of dendritic cell activity in controls, moderate and severe CFS/ME patients using CD80 and CD86 activation markers. DC activity is shown based on the difference in expression of activation markers (CD80 and CD86) following stimulation. Measures are represented as percentage of DCs expressing markers (\%) in controls, moderate CFS/ME and severe CFS/ME. All data are represented as mean \pm SEM. CFS/ME: Chronic Fatigue Syndrome/Myalgic Encephalomyelitis, DC: Dendritic Cell. SEM: Standard Error of the Mean.

Additional file 2: Figure S2. Flow cytometric analysis of monocyte and neutrophil function in controls, moderate and severe CFS/ME patients. A Monocyte and Neutrophil function respiratory burst, shown as difference in mean fluorescence intensity following stimulation. Measures are shown for controls, moderate CFS/ME and severe CFS/ME. B Monocyte and Neutrophil function phagocytosis, shown as difference in mean fluorescence intensity following stimulation. Measures are shown for controls, moderate CFS/ME and severe CFS/ME. CFS/ME: Chronic Fatigue Syndrome/Myalgic Encephalomyelitis.

Additional file 3: Figure S3. Intracellular analysis of lytic proteins in controls, moderate and severe CFS/ME patients. A Lytic proteins in iNKT cells are shown as percentage of iNKT cells (\%) expressing perforin, granzyme A, granzyme B and CD57 for controls, moderate CFS/ME and severe CFS/ME patients. B Lytic proteins in Tregs are shown as percentage of Tregs (\%) expressing the lytic proteins perforin, granzyme $A$, granzyme B and CD57 in controls, moderate CFS/ME and severe CFS/ ME patients. All data are represented as mean \pm SEM. Chronic Fatigue Syndrome/Myalgic Encephalomyelitis; Treg: T Regulatory Cell. SEM: Standard Error of the Mean.

Additional file 4: Figure S4. Profile of Bregs and BCRs in controls, moderate and severe CFS/ME patients. A Bregs are as percentage of $\mathrm{B}$ cells (\%) in controls, moderate CFS/ME and severe CFS/ME patients. $B C D 79 b$ and IgD expression to represent BCRs and shown as a percentage of $B$. BCRs shown in controls, moderate CFS/ME and severe CFS/ME patients. All data are represented as mean \pm SEM. Breg: B Regulatory Cell; BCR: B Cell Receptor; Chronic Fatigue Syndrome/ Myalgic Encephalomyelitis; Treg: T Regulatory Cell. SEM: Standard Error of the Mean.

Additional file 5: Figure S5. Peripheral blood $C D 4^{+} T$ cell phenotypes in controls, moderate and severe CFS/ME patients. CD45RA effector memory, naïve, central memory and effector memory $C D 4^{+} T$ cell phenotypes represented as percentage of total $\mathrm{CD} 4^{+} \mathrm{T}$ cells (\%). All data are represented as mean \pm SEM. CFS/ME: Chronic Fatigue Syndrome/ Myalgic Encephalomyelitis; SEM: Standard Error of the Mean.

Additional file 6: Table S1. Monoclonal antibody combinations used to identify various innate and adaptive immune cells and phenotypes.

\section{Abbreviations}

ANOVA: Analysis of variance test; $B C R$ : $B$ cell receptor; Breg: $B$ regulatory cell; BTLA: B and T lymphocyte attenuator; CFS: Chronic fatigue syndrome; DC: Dendritic cell; DHR: Dihydrohodamine; EDTA: Ethylenediaminetetraacetic acid; KPS: Karnofsky performance scale; FSS: Fatigue severity scale; HIV: Human immunodeficiency syndrome; IFN: Interferon; IL: Interleukin; KIR: Killer immunoglobulin-like receptor; KLRG: Killer cell lectin-like receptor subfamily G member; LFA: Lymphocyte function-associated antigen; MDD: Major depressive disorder; ME: Myalgic Encephalomyelitis; MHC: Major histocompatibility complex; MS: Multiple sclerosis; NCR: Natural cytotoxicity receptor; NK: Natural killer; NFAT: Nuclear factor of activated t cells; PBMC: Peripheral blood mononuclear cell; PMA: Phorbol 12-myristate 13acetate; RPMI: Roswell park memorial institute; SAP: SLAM associated protein; SD: Standard deviation; SEM: Standard error of the mean; SLAM: Signaling lymphocytic activation molecule; SLE: Systemic lupus erythematosus; TNF: Tumour necrosis factor; Treg: T regulatory cell; $ү \delta:$ Gamma delta.

\section{Competing interests}

The authors declare that they have no competing interests.

\section{Authors' contributions}

SH participated in the study design, patient recruitment, laboratory analysis, statistical analysis and drafted the manuscript. EB, SJ, SR, DS and SMG participated in the study design, patient coordination, data interpretation and drafting of the manuscript. NW, TN and TH assisted in the collection and analysis of laboratory data and revised the manuscript. All authors read and approved the final manuscript.

\section{Acknowledgements}

Alison Hunter Memorial Foundation, Mason Foundation [Grant Number MA43120] and Queensland Government Department of Science, Information Technology, Innovation and the Arts Smart Futures Fund [Grant Number 216702MRE].

Received: 28 November 2014 Accepted: 21 May 2015 Published online: 02 June 2015

\section{References}

1. Maes $M$, Twisk FN, Kubera M, Ringel K. Evidence for inflammation and activation of cell-mediated immunity in myalgic encephalomyelitis/chronic fatigue syndrome (ME/CFS): increased interleukin-1, tumor necrosis factor-a, PMN-elastase, lysozyme and neopterin. J Affect Disord. 2012;136(3):933-9.

2. Seide A, Arolt V, Hunstiger M, Rink L, Behnisch A, Kirchner H. Increased CD56 $<$ sup $>+</$ sup $>$ natural killer cells and related cytokines in major depression. Clin Immunol Immunopathol. 1996;78(1):83-5.

3. Blanca IR, Bere EW, Young HA, Ortaldo JR. Human B cell activation by autologous NK cells is regulated by CD40-CD40 ligand interaction: role of memory B cells and CD5+ B cells. J Immunol. 2001;167(11):6132-9.

4. Schepis D, Gunnarsson I, Eloranta ML, Lampa J, Jacobson SH, Kärre K, et al. Increased proportion of CD56bright natural killer cells in active and inactive systemic lupus erythematosus. Immunology. 2009;126(1):140-6.

5. Hardcastle S, Brenu E, Johnston S, Nguyen T, Huth T, Kaur M. Analysis of the relationship between immune dysfunction and symptom severity in patients with Chronic Fatigue Syndrome/Myalgic Encephalomyelitis (CFS/ ME). J Clin Cell Immunol. 2014;5(190):2.

6. Landay A, Lennette E, Jessop C, Levy J. Chronic fatigue syndrome: clinical condition associated with immune activation. Lancet. 1991;338(8769):707-12.

7. Brenu, Hardcastle, Atkinson G, van Driel M, Kreijkamp-Kaspers S, Ashton K, et al.: Natural killer cells in patients with severe chronic fatigue syndrome. Autoimmunity Highlights 2013;4(3):1-12.

8. Brenu EW, Staines DR, Baskurt OK, Ashton KJ, Ramos SB, Christy RM, et al. Immune and hemorheological changes in chronic fatigue syndrome. J Transl Med. 2010;8(1):1. 
9. Brenu EW, van Driel ML, Staines DR, Ashton KJ, Hardcastle SL, Keane J, et al. Longitudinal investigation of natural killer cells and cytokines in chronic fatigue syndrome/myalgic encephalomyelitis. J Transl Med. 2012;10:88.

10. Brenu EW, van Driel ML, Staines DR, Ashton KJ, Ramos SB, Keane J, et al. Immunological abnormalities as potential biomarkers in chronic fatigue syndrome/myalgic encephalomyelitis. J Transl Med. 2011;9:81.

11. Klimas NG, Salvato FR, Morgan R, Fletcher MA. Immunologic abnormalities in chronic fatique syndrome. J Clin Microbiol. 1990;28(6):1403-10.

12. Levine PH, Whiteside TL, Friberg D, Bryant J, Colclough G, Herberman RB. Dysfunction of natural killer activity in a family with chronic fatigue syndrome. Clin Immunol Immunopathol. 1998;88(1):96-104.

13. Ojo-Amaize EA, Conley EJ, Peter JB. Decreased natural killer cell activity is associated with severity of chronic fatigue immune dysfunction syndrome. Clin Infect Dis. 1994;18(Supplement 1):S157-9.

14. Patarca-Montero R, Antoni M, Fletcher MA, Klimas NG. Cytokine and other immunologic markers in chronic fatigue syndrome and their relation to neuropsychological factors. Appl Neuropsychol. 2001;8(1):51-64.

15. Barker E, Fujimura SF, Fadem MB, Landay AL, Levy JA. Immunologic abnormalities associated with chronic fatigue syndrome. Clin Infect Dis. 1994;18(Supplement 1):S136-41

16. Brenu E, Johnston S, Hardcastle S, Huth T, Fuller K, Ramos S, et al. Immune abnormalities in patients meeting new diagnostic criteria for chronic fatigue syndrome/Myalgic Encephalomyelitis. J Mol Biomark Diagn. 2013:4(152.10):4172

17. Brenu EW, Huth TK, Hardcastle SL, Fuller K, Kaur M, Johnston S, et al. Role of adaptive and innate immune cells in chronic fatigue syndrome/myalgic encephalomyelitis. Int Immunol. 2013;26:dxt068.

18. Brenu EW, van Driel M, Staines DR, Kreijkamp-Kaspers S, Hardcastle SL, Marshall-Gradisnik SM. The effects of influenza vaccination on immune function in patients with Chronic Fatigue Syndrome/Myalgic Encephalomyelitis. International J Clinical Med. 2012;3:544

19. Caligiuri M, Murray $C$, Buchwald D, Levine $H$, Cheney $P$, Peterson D, et al. Phenotypic and functional deficiency of natural killer cells in patients with chronic fatigue syndrome. J Immunol. 1987;139(10):3306-13.

20. Maher KJ, Klimas NG, Fletcher MA. Chronic fatique syndrome is associated with diminished intracellular perforin. Clin Exp Immunol. 2005;142(3):505-11.

21. Patarca R, Klimas NG, Garcia MN, Walters MJ, Dombroski D, Pons H, et al. Dysregulated expression of soluble immune mediator receptors in a subset of patients with chronic fatigue syndrome: cross-sectional categorization of patients by immune status. J Chronic Fatigue Syndrome. 1995;1(1):81-96.

22. Tirelli U, Marotta G, Improta S, Pinto A. Immunological abnormalities in patients with chronic fatigue syndrome. Scand J Immunol. 1994;40(6):601-8.

23. Aoki T, Miyakoshi H, Usuda Y, Herberman RB. Low NK syndrome and its relationship to chronic fatigue syndrome. Clin Immunol Immunopathol. 1993;69(3):253-65

24. Huth T, Brenu E, Nguyen T, Hardcastle S, Johnston S. Characterization of natural killer cell phenotypes in chronic fatigue Syndrome/Myalgic Encephalomyelitis. J Clin Cell Immunol. 2014;5(223):2.

25. Brenu EW, Ashton KJ, van Driel M, Staines DR, Peterson D, Atkinson GM, et al. Cytotoxic lymphocyte microRNAs as prospective biomarkers for chronic fatigue syndrome/myalgic encephalomyelitis. J Affect Disord. 2012;141(2):261-9.

26. Saiki T, Kawai T, Morita K, Ohta M, Saito T, Rokutan K, et al. Identification of marker genes for differential diagnosis of chronic fatique syndrome. Mol Med. 2008;14(9-10):599.

27. Baraniuk JN, Adewuyi O, Merck SJ, Ali M, Ravindran MK, Timbol CR, et al. A Chronic Fatigue Syndrome (CFS) severity score based on case designation criteria. Am J Transl Res. 2013;5(1):53-68.

28. Carruthers BM, van de Sande MI, De Meirleir KL, Klimas NG, Broderick G, Mitchell T, et al. Myalgic encephalomyelitis: international consensus criteria. J Intern Med. 2011:270(4):327-38.

29. Fukuda K, Straus SE, Hickie I, Sharpe MC, Dobbins JG, Komaroff A. The chronic fatigue syndrome: a comprehensive approach to its definition and study. Ann Intern Med. 1994;121(12):953-9.

30. Zaturenskaya M, Jason LA, Torres-Harding S, Tryon WW. Subgrouping in chronic fatigue syndrome based on actigraphy and illness severity. Open Biology J. 2009;2:20-6.

31. Jason LA, Corradi K, Torres-Harding S, Taylor RR, King C. Chronic fatigue syndrome: the need for subtypes. Neuropsychol Rev. 2005;15(1):29-58.

32. Wiborg JF, van der Werf S, Prins JB, Bleijenberg G. Being homebound with chronic fatigue syndrome: a multidimensional comparison with outpatients. Psychiatry Res. 2010;177(1):246-9.
33. Lloyd AR, Wakefield D, Boughton C, Dwyer J. Immunological abnormalities in the chronic fatigue syndrome. Med J Aust. 1989;151(3):122.

34. Bansal A, Bradley A, Bishop K, Kiani-Alikhan S, Ford B. Chronic fatigue syndrome, the immune system and viral infection. Brain Behav Immun. 2012;26(1):24-31.

35. Fluge $\varnothing$, Bruland $O$, Risa K, Storstein A, Kristoffersen EK, Sapkota D, et al. Benefit from B-lymphocyte depletion using the anti-CD20 antibody rituximab in chronic fatigue syndrome. A double-blind and placebo-controlled study. PLoS One. 2011;6(10):e26358.

36. Klimas NG, Broderick G, Fletcher MA. Biomarkers for chronic fatigue. Brain Behav Immun. 2012;26(8):1202-10.

37. Sidorenko SP, Clark EA. The dual-function CD150 receptor subfamily: the viral attraction. Nat Immunol. 2003;4(1):19-24.

38. Lanier LL. On guard-activating NK cell receptors. Nat Immunol. 2001;2(1):23-7.

39. Wang N, Morra M, Wu C, Gullo C, Howie D, Coyle T, et al. CD150 is a member of a family of genes that encode glycoproteins on the surface of hematopoietic cells. Immunogenetics. 2001;53(5):382-94.

40. Veillette A. NK cell regulation by SLAM family receptors and SAP-related adapters. Immunol Rev. 2006;214(1):22-34.

41. Biassoni R, Cantoni C, Pende D, Sivori S, Parolini S, Vitale M, et al. Human natural killer cell receptors and co-receptors. Immunol Rev. 2001:181(1):203-14.

42. Poli $A$, Michel $T$, Thérésine $M$, Andrès $E$, Hentges $F$, Zimmer J. CD56bright natural killer (NK) cells: an important NK cell subset. Immunology. 2009;126(4):458-65.

43. Anane LH, Edwards KM, Burns VE, Zanten JJ, Drayson MT, Bosch JA. Phenotypic characterization of $\gamma \delta$ T cells mobilized in response to acute psychological stress. Brain Behav Immun. 2010;24(4):608-14.

44. Sallusto F, Geginat J, Lanzavecchia A. Central memory and effector memory $T$ cell subsets: function, generation, and maintenance. Annu Rev Immunol. 2004:22:745-63.

45. Farber DL, Yudanin NA, Restifo NP. Human memory T cells: generation, compartmentalization and homeostasis. Nat Rev Immunol. 2014;14(1):24-35.

46. Henson SM, Akbar AN. KLRG1 - more than a marker for T cell senescence. Age. 2009;31(4):285-91

47. Blackburn SD, Shin H, Haining WN, Zou T, Workman CJ, Polley A, et al. Coregulation of CD8+ T cell exhaustion by multiple inhibitory receptors during chronic viral infection. Nat Immunol. 2008;10(1):29-37.

48. Curriu M, Carrillo J, Massanella M, Rigau J, Alegre J, Puig J, et al. Screening NK-B-and T-cell phenotype and function in patients suffering from Chronic Fatigue Syndrome. J Transl Med. 2013;11:68.

49. Estefanía E, Flores R, Gómez-Lozano N, Aguilar H, López-Botet M, Vilches C. Human KIR2DL5 is an inhibitory receptor expressed on the surface of NK and T lymphocyte subsets. J Immunol. 2007;178(7):4402-10.

50. Cisneros E, Moraru M, Gómez-Lozano N, López-Botet M, Vilches C. KIR2DL5: an orphan inhibitory receptor displaying complex patterns of polymorphism and expression. Front Immunol. 2012;3.

51. Vilches $C$, Rajalingam $R$, Uhrberg M, Gardiner CM, Young NT, Parham P. KIR2DL5, a novel killer-cell receptor with a D0-D2 configuration of Ig-like domains. J Immunol. 2000;164(11):5797-804.

52. Hurchla MA, Sedy JR, Murphy KM. Unexpected role of B and T lymphocyte attenuator in sustaining cell survival during chronic allostimulation. J Immunol. 2007;178(10):6073-82.

53. Nielsen N, Ødum N, Ursø B, Lanier LL, Spee P. Cytotoxicity of CD56bright NK cells towards autologous activated CD4+ T cells is mediated through NKG2D, LFA-1 and TRAIL and dampened via CD94/NKG2A. PLoS One. 2012;7(2), e31959.

54. Bryceson YT, March ME, Ljunggren HG, Long EO. Activation, coactivation, and costimulation of resting human natural killer cells. Immunol Rev. 2006;214(1):73-91.

55. Lima M, Almeida J, dos Anjos TM, Queirós ML, Justiça B, Orfão A. The " $<\mathrm{i}>$ ex Vivo</i>" Patterns of CD2/CD7, CD57/CD11c, CD38/CD11b, CD45RA/ CD45RO, and CD11a/HLA-DR Expression Identify Acute/Early and Chronic/ Late NK-Cell Activation States. Blood Cell Mol Dis. 2002;28(2):181-90.

56. Glaser R, Kiecolt-Glaser JK. Stress-induced immune dysfunction: implications for health. Nat Rev Immunol. 2005;5(3):243-51.

57. Bryant PA, Trinder J, Curtis N. Sick and tired: does sleep have a vital role in the immune system? Nat Rev Immunol. 2004;4(6):457-67.

58. Üstün TB. Measuring health and disability: Manual for WHO disability assessment schedule WHODAS 2.0. In: World Health Organization. 2010. 
59. Ware Jr JE, Sherbourne CD. The MOS 36-item short-form health survey (SF-36): I. Conceptual framework and item selection. In: Medical care. 1992. p. 473-83.

60. Montoya CJ, Pollard D, Martinson J, Kumari K, Wasserfall C, Mulder CB, et al. Characterization of human invariant natural killer T subsets in health and disease using a novel invariant natural killer T cell-clonotypic monoclona antibody, 6B11. Immunology. 2007;122(1):1-14.

61. Aguinis H, Gottfredson RK, Joo H. Best-practice recommendations for defining, identifying, and handling outliers. Organ Res Methods. 2013;16(2):270-301.

Submit your next manuscript to BioMed Central and take full advantage of:

- Convenient online submission

- Thorough peer review

- No space constraints or color figure charges

- Immediate publication on acceptance

- Inclusion in PubMed, CAS, Scopus and Google Scholar

- Research which is freely available for redistribution 